\title{
Impact of a tree's hydraulic strategy on its survival in a global climate change context
}

\author{
$\underline{\text { J. Chopard }}^{\text {a,b }}$, M. Bader ${ }^{\text {a,b }}$, E.J. Veneklaas ${ }^{\text {a,b }}$ and M. Renton ${ }^{\text {a,b,c }}$ \\ ${ }^{a}$ Centre of Excellence for Climate Change Woodland and Forest Health, University of Western Australia \\ ${ }^{b}$ School of Plant Biology, The University of Western Australia \\ ${ }^{c}$ CSIRO Ecosystem Sciences \\ Email: jerome.chopard@uwa.edu.au
}

\begin{abstract}
Global climate models predict less rain for many regions with Mediterranean climates such as the South West of Western Australia, together with an increasing frequency of extreme events (e.g. exceptionally warm and dry years or days). In this context it is important to understand how trees in natural environments will respond to changing conditions. Over millions of years, evolution has favoured different strategies to enable these plants to extract water at a rate sufficient to sustain themselves even in these relatively arid regions where summer drought is a regular occurrence. These strategies involve mechanisms occurring at the cell scale, such as stomatal regulation, increased vessel size to improve the conductance for water flow or increased elasticity of cell walls to increase water capacitance of sapwood. These mechanisms all have interrelated effects and there are important trade-offs between them. For example, increasing the conductance of vessels by increasing the size of pit pores between vessel elements also leads to a greater susceptibility to embolism and hence an increased risk of complete loss of conductivity in the case of a particularly extreme drought. Growing new roots to facilitate water uptake requires a significant investment of carbon that must be obtained by photosynthesis at the expense of more transpired water.
\end{abstract}

We need a model to understand the importance of cell-level changes for the adaptation of trees to their environment. At the interface between the microscopic scale of plant cells and the macroscopic scale of the vegetation layer used in climate models, a model of the hydraulics of a single individual tree can be used to test different strategies for water uptake, transport and transpiration. In this paper we will describe how we developed such a model (Figure 1), how we used the model to analyse some overall strategies adopted by trees and discuss them in the light of drought tolerance. Results show that optimizations for water use occurs at different scales and are interrelated. At long time scale, trees can adapt the allocation of carbon to different parts of their architecture (roots, trunk, and canopy) to keep their stomata open longer. Within a day, stomatal control prevents hydraulic failures and adjusted tissue water capacitance allows the tree to answer rapid changes in physical conditions.
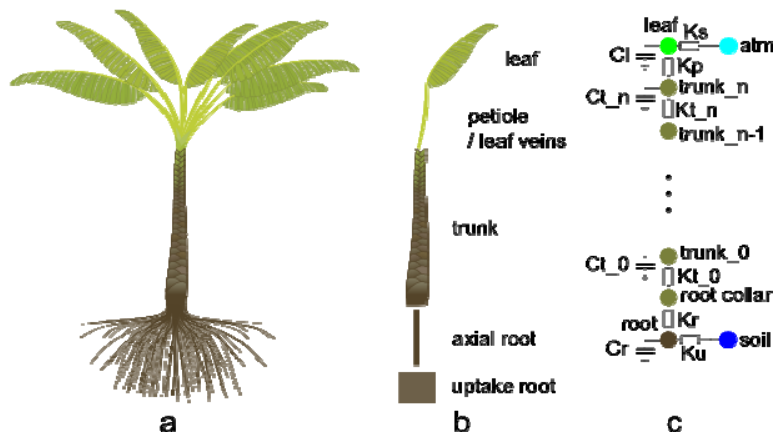

Figure 1. Architecture of a palm tree: a) whole plant, b) functional compartments used in the model and c) electric analogy of an hydraulic representation of the plant (circles represents compartments characterized by a uniform water potential, boxes stands for the hydraulic conductance $(K)$ between two compartments and capacitors $(C)$ represent water storage).

Keywords: Darcy's law, embolism, stomata closure, plant hydraulic modelling 


\section{INTRODUCTION}

A tree's growth and survival depend on its capacity to perform gas exchange with the atmosphere, i.e. gain $\mathrm{CO}_{2}$ at the expense of losing water through its leaves. This capacity depends on the interaction of different parts of the plant that can be summarized as:

- A root system for water uptake from the soil;

- A transpiring leafy canopy;

- A trunk that connects the two previous components and stores some water to buffer rapid changes in atmospheric conditions.

The interactions between these components are complex and encompass different scales. To understand the benefit of a given adaptation and predict the survival of a given strategy in changing conditions, a model of tree water relations is required.

\section{MODEL DESCRIPTION}

The model presented in this article was developed to help understand how different strategies used by different parts of the plant lead to maximizing gas exchange through stomatal opening. Growth or carbon production components have initially been omitted to keep the model simple enough to perform the analyses but will be implemented at advanced model stages.

\subsection{Plant architecture}

For this study, we chose a general simplified palm-tree-like architecture (see Figure 1). The main parameters of this architecture are the total leaf surface area, the total root uptake surface area and the size of the trunk. All other parameters can be derived from these three basic structural parameters assuming some fixed geometrical constants. This approach simplifies the exploration of different strategies, keeping the shape factors constant and unravelling the effect of the functional parameters only.

In this representation, each leaf is a simple rectangular shape characterized by its length, width and thickness. Since plant growth is not part of the model, these parameters will be treated as constants during a simulation. For a given total leaf surface area, we can then estimate the number of leaves in the tree and the total volume of the leaf compartment.

The root system is composed of main axes connected to the trunk and supporting small rootlets where uptake actually occurs. As for the canopy, we will consider that the geometry of each absorbing rootlet is fixed (cylinder of given length and radius). For a given total uptake surface area, we can then estimate the number of main axes needed to support it and the volume of the root compartment (approximated by the volume of the main axes only).

\subsection{Hydraulic description}

Water flows through the plant down a gradient of water potential from high values in the soil to low values in the atmosphere. Along this path, each compartment of the plant will oppose a certain resistance to the flow. Darcy's law states that the flow through a given compartment can be adequately modelled as:

\section{$l_{u \rightarrow d}=K\left(\psi_{u}-\psi_{d}\right)$}

where $\psi_{u}, \psi_{d}$ in MPa are the water potential upstream and downstream of the compartment respectively, $J_{u \rightarrow a}$ is the flow of water in $\mathrm{m}^{3} \mathrm{~s}^{-1}$ and $K$ is the conductance in $\mathrm{m}^{3} \mathrm{~s}^{-1} \mathrm{MPa}^{-1}$ (inverse of resistance) of the compartment. Along its path, water has to cross different parts of the plant:

- Root cortex to enter the plant from the soil

- Root axis to travel from the uptake zone to the root collar at the base of the trunk of the plant

- Trunk axis to reach the canopy

- The petiole and leaf veins connecting the top of the trunk to the inside of a leaf

- Stomata, which provide a final barrier to the flux of water from inside the leaf to the atmosphere

Each of these compartments, except for the stomata (see below), is characterized by a fixed conductivity.

$k=k \frac{s}{l}$ 
$K$ is the conductance of the compartment $\left(\mathrm{m}^{3} \mathrm{~s}^{-1} \mathrm{MPa}^{-1}\right), k$ its conductivity $\left(\mathrm{m}^{2} \mathrm{~s}^{-1} \mathrm{MPa}^{-1}\right), S$ the surface area $\left(\mathrm{m}^{2}\right)$ through which water flows and $l$ the distance $(\mathrm{m})$ water has to travel to cross the compartment.

Water is also stored in compartments whose volume is not negligible: root main axes, trunk and leaf blade. The amount of water available depends on the elasticity of the cells that store the water. A simple formulation (Tyree, 1990) considers that the amount of water $\left(Q \mathrm{in}^{3}\right)$ that can be extracted from a storage compartment is linearly proportional to the water potential in the compartment:

$Q=C \psi$ with $C=c V$

$C$ is the capacitance of the compartment in $\mathrm{m}^{3} \mathrm{MPa}^{-1}$ that can be expressed as the product of the specific capacity $c$ in $\mathrm{m}^{3} \mathrm{~m}^{-3} \mathrm{MPa}^{-1}$ by the actual volume of the compartment $V$ in $\mathrm{m}^{3}$.

\subsection{Soil and Atmosphere}

Water potential in the atmosphere $\left(\psi_{\mathrm{L}} \mathrm{sm}\right)$ is related to temperature $(T$ in $\mathrm{K})$ and relative humidity $(H)$ :

$\psi_{\mathrm{atm}}=\frac{B}{M_{W}} T \log R$

where $R$ is the perfect gas constant $\left(\mathrm{J} \mathrm{mol}^{-1} \mathrm{~K}^{-1}\right)$ and $M_{w}$ is the molar mass of water $\left(\mathrm{kg} \mathrm{mol}{ }^{-1}\right)$. On a typical winter day in Perth, Western Australia, water potential in the atmosphere will range from around 0MPa by night to $-100 \mathrm{MPa}$ in the middle of the day.

Water potential in the soil depends on the soil water content and soil texture and structure (particle size distribution and pore sizes). This relationship is summarized in a water retention curve that can be measured for different type of soils (Smettem, 1996).

\subsection{Stomatal control}

As will be demonstrated below, plants have to regulate the aperture of their stomata to increase gas exchange with the atmosphere while preventing the water potential in the plant to drop below a certain threshold leading to permanent loss of conductivity through embolism (bubbles of air entering the vessels). Different kinds of models have been proposed to link stomata aperture to environmental conditions of light, temperature and humidity. In our model, we use a simple formulation where plants tend to close their stomata by night and also as soon as the water potential in the leaf ( $\left.\psi_{l}\right)$ drops below a given threshold ( $\left.\psi_{t h}\right)$. Opening and closure are physiological processes that require some time, so $\sigma_{o}$ represents the rate of opening $\left(\mathrm{s}^{-1}\right)$ and $\alpha_{0}$ represents the rate of closure $\left(\mathrm{s}^{-1}\right)$. The overall stomata conductance $\left(K_{s}\right)$ model is:

$\frac{\partial K_{z}}{\partial t}= \begin{cases}-\alpha_{g} K_{g}, & \psi_{z} \psi_{t h} \text { or during the night } \\ \alpha_{g} K_{z}, & \psi_{z} z \psi_{t h} \text { only during daylight }\end{cases}$

\subsection{Numerical methods}

A finite element approach has been taken to compute the hydraulic state of the tree at a given stage. The tree architectural compartments are divided in a limited number of elements characterized by a uniform water potential. The actual geometry of each element is inferred from the main overall parameters of the tree (leaf surface area, trunk size, root uptake area). The conductance and capacitance of each element is computed from the dimensions of the element using parameters summarized in Table 1 (Appendix 1).

Each individual equation presented above is linear and can be solved using a fully implicit scheme. This approach ensures convergence even for big time steps. However, since the stomata closure model is based on a threshold, to choose a big time step leads to overcompensations by stomata (i.e. stomata continue to close/open even after the condition is no longer valid). For simulations that require a precise control of stomata, we choose a time step equals to a hundredth of $\frac{1}{\alpha_{o}}$ and $\frac{1}{\alpha_{c}}$.

\section{HYDRAULIC STRATEGIES}

The model presented in the previous section is general enough to account for a wide range of environments and plant parameters and simple enough to allow exploration of the influence of some parameters on the water taken up by plants. In this part, we intend to have a look at some of the strategies used by plants to optimize their water uptake. 


\subsection{Operating water potential and embolism}

The total amount of water a plant can extract from the soil is defined as the difference between soil water content at field capacity and at the water potential corresponding to the lowest root water potential. Therefore, it seems beneficial for the plant to achieve extremely low water potential in its roots. There is a trade-off between achieving low water potential and maintaining plant water uptake throughout time.

Low water potentials are usually associated with embolisms which break the water column and lead to a partial loss of conductivity. This effect is summarized in the measured vulnerability curves that represent the amount of conductivity loss as a function of water potential in an element (see Figure 2 (a) ). The model can be used to compute the evolution of water potential in a given part of the plant when the conductivity of this part varies. Figure 2 (b) shows such curves for different imposed values of stomata opening.
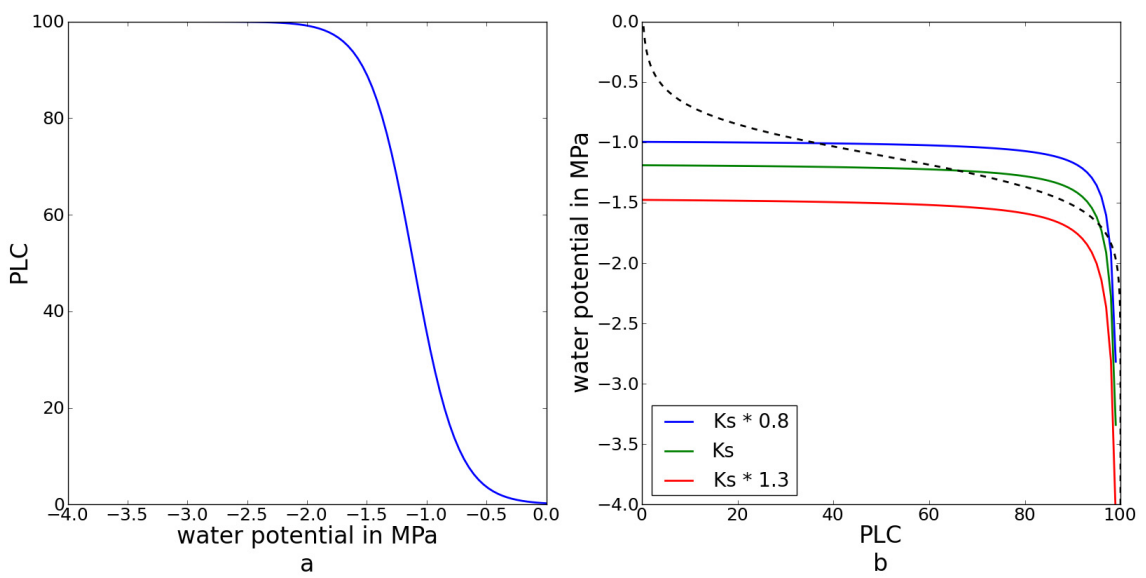

Figure 2. a) Typical measured vulnerability curve, percentage loss of conductance (PLC) as a function of branch water potential. b) Simulation of branch water potential as a function of loss of conductance in the branch. Each curve represents a different stomatal conductance $\left(\mathrm{K}_{\mathrm{s}}\right)$. The vulnerability curve has been superimposed as a dotted line.

The comparison of the two sets of curves shows that two situations can occur:

- a stable partial embolism characterized by a limited loss of conductance proportional to the water potential in the leaf (Figure 3 (a) );

- a runaway embolism characterized by a complete loss of conductance for the given element as soon as the water potential in the leaf falls below a certain threshold (Figure 3 (b) ).
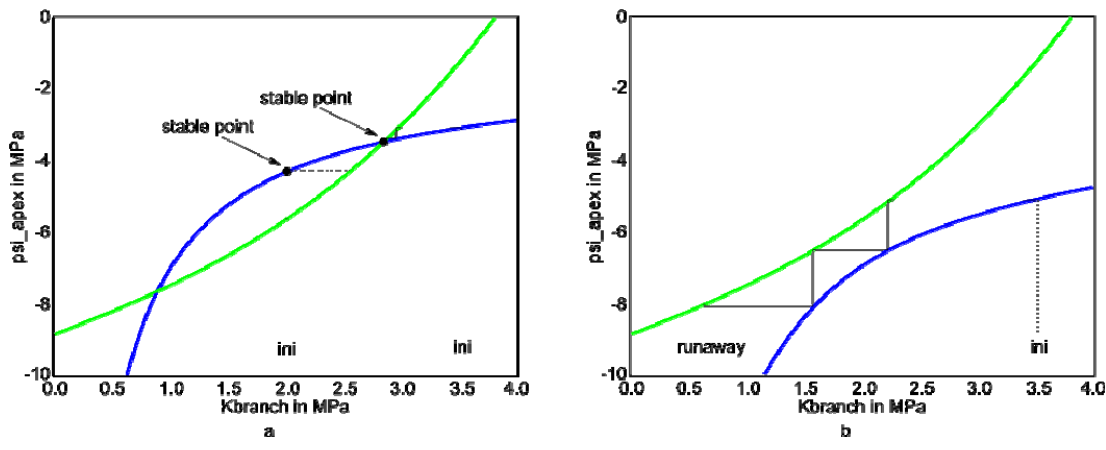

Figure 3. Spread of embolism. Superposition of the vulnerability curve (green) with the curve (blue) representing the evolution of water potential in the branch as a function of branch conductance. a) Stable: starting from an initial high conductance of the branch (ini), the blue curve reads the corresponding water potential in the branch. This water potential is then used to read the amount of conductance lost by the branch (on the green curve). Eventually this cycle leads to a stable point whatever the 'ini' point chosen. b) Runaway embolism: whatever the initial branch conductance (ini), there is no stable point and branch conductance drops to zero. 
In order to avoid runaway embolism in the important parts of its architecture (e.g. trunk or main branches), plants must maintain the water potential in their leaves above a certain threshold by closing their stomata.

\subsection{Ratio leaves / roots}

The capacity of a tree to uptake water from the soil and ultimately to fix carbon depends on the amount of carbon it invests in its transport and storage structure. If the water potential in the plant were not limited by embolism, all allocation strategies would be similar from a water point of view. However, since plants cannot operate below a given water potential threshold, the amount of water flowing through a plant strongly depends on the partitioning of carbon between the different compartments.

If we consider that the amount of structural carbon in a compartment is proportional to the volume of this compartment, the model can be used to predict water uptake by the plant for different allocation strategies.
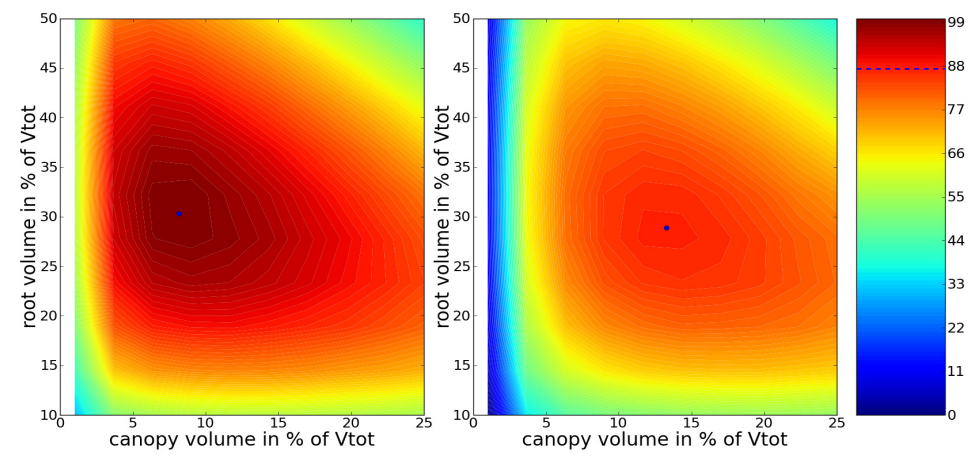

Figure 4. Carbon allocation strategy for two leaf thicknesses. Leaves on the right simulation are three times as thick as the leaves on the left. Colour maps display the efficiency of allocating more carbon to one compartment in terms of keeping stomata as open as possible for as long as possible. Efficiencies are rated from 0 for the worst to $100 \%$ for the best of the two simulations. Blue dots on each map mark the exact position of the best strategy. The dotted line on the colour bar marks the efficiency of the best strategy for thick leaves (on the right) compared to slim leaves (on the left). In both simulations, trunk volume is computed to keep the total volume of the tree constant.

Figure 4 presents the result of such computation where the total amounts of carbon have been kept constant. The simulations show that for a given set of structural parameters (leaf thickness and root density) there is a unique optimum that maximize stomata opening throughout time. A comparison of the two simulations shows that investing carbon to increase leaf thickness is not an efficient strategy to maintain stomata open longer.

\subsection{Tissue capacitance}

We have seen above that plants have to operate above a water potential threshold and that some allocation strategies are more efficient to maintain their stomata open. Strong stomatal control ensures that leaf water potential will stay above the critical threshold. However, being a physiological process, stomata closure is not potential will stay

instantaneous and is characterized by a typical time, $\overline{\alpha_{\varepsilon}}$, of the order of minutes (Losch 1977). If the plant typically experiences physical conditions varying within a short time (e.g. wind blow dry instead of humid air around the leaf for a few seconds), the stomatal response will not be quick enough to prevent leaf water potential from dropping below the critical threshold (see Figure 5 green curve). To avoid such catastrophic events, a plant can operate at a water potential above the critical threshold giving a security margin (see Figure 5 blue curve). The size of this margin depends on the frequency and amplitude of such short-term events. 


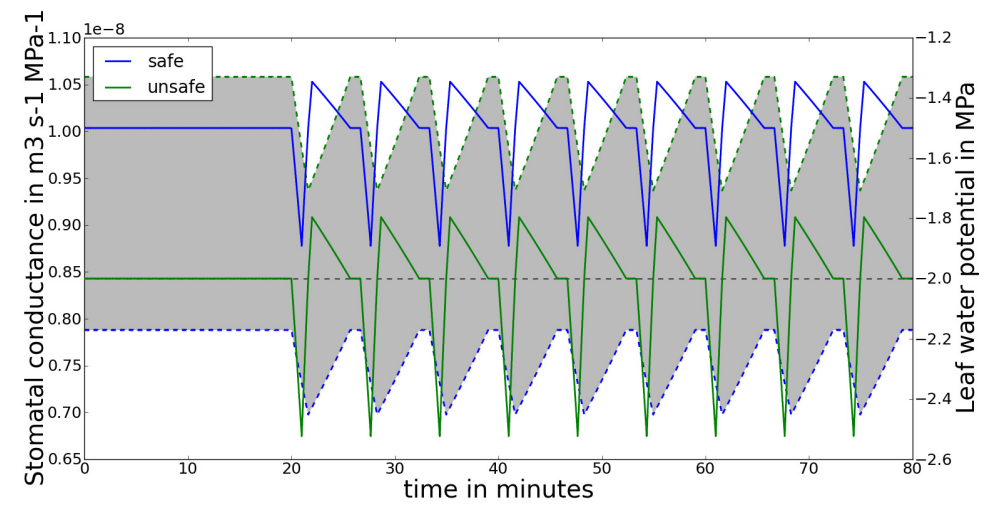

Figure 5. Atmospheric perturbations. The graph shows the effect of sudden drops (10\% of relative humidity) of atmospheric water potential on stomatal conductance (dotted lines) and leaf water potential (plain lines). The perturbations start at 20 minutes and repeats every 6 minutes. The horizontal black dotted line represents the leaf water potential threshold below which runaway embolism occurs. The gray area emphasizes the difference between the stomatal openings of both strategies.

To operate at a higher water potential on average, leaves have to close their stomata a little bit more (Figure 5 , grey area). This strategy is therefore not optimal. Another strategy is to let leaves store some water to buffer the effect of perturbation events. Figure 6 shows the evolution of leaf water potential around a perturbation for different buffer configurations. The characteristic time associated with the discharge of the buffer is equal to the ratio of the capacitance of the buffer divided by the conductance of stomata. Hence, the bigger the buffer, the longer the characteristic time and the smaller the variation of water potential a leaf experiences for a given perturbation (Figure 6, curves red and green).

Increasing the leaf capacitance is limited by the fact that increasing leaf volume (allocating more carbon to leaves) without increasing the volume of the other tree compartments is not an efficient strategy for water uptake as has been demonstrated above. Moreover, the time needed to refill the buffer is proportional to the capacitance of the buffer divided by the overall conductance of the tree between the leaf and the soil. Therefore, having a bigger leaf capacitance reduces the drop in water potential but increases the time the water potential stay above the optimal threshold afterward. Simulations (Figure 6, light blue curve) show that a more efficient strategy is keeping only a small capacitance in the leaves to buffer the effects of perturbations and connect this to a bigger capacitance in the trunk to refill the leaf buffer quickly.

\section{CONCLUSIONS}

In this article we have demonstrated that a simple model of tree water relations can be used to investigate the

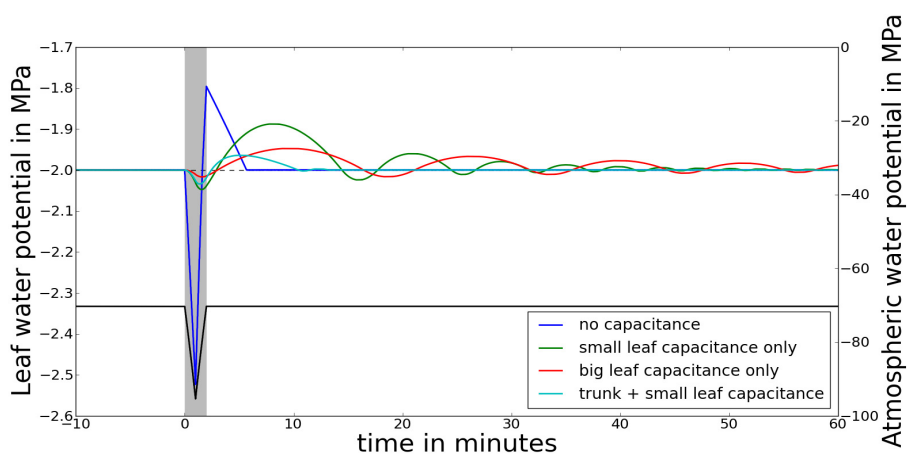

Figure 6. Role of tissue capacitance. The graph shows the effect of a single atmospheric perturbation (black line), similar to the one used in Figure 5 (10\% drop of relative humidity in one minute), applied to a tree for different combination of capacitance. Duration of the perturbation in the atmosphere has been highlighted in grey. 
effects of different strategies on the capacity of the tree to keep its stomata open and ultimately its survival. These strategies encompass different scales:

- Leaf level with a precise control of the operating leaf water potential through stomatal regulation and leaf capacitance

- Organ level with a trade-off between conductance and vulnerability to embolism

- Whole tree level with different strategies of allocation of carbon to root, leaves or trunk reflecting different performances in terms of water uptake

The need for such a model is further enhanced by the added complexity that all these strategies are interrelated.

We intend to use this model to estimate tree survival under global climate change scenarios. Different physical conditions will require adaptations in the tree to prevent hydraulic failure, or will lead to outperformance of certain strategies by other more efficient ones. The model will be used to estimate the range of change required under certain climate projections and help us estimate if real trees in the field can operate in this range and survive drier and hotter climates.

\section{REFERENCES}

Losch R. (1977). Responses of stomata to environmental factors - experiments with isolated epidermal strips of Polydium vulgare. Oecologia, 29, 85-97.

Smettem K. and Gregory P. (1996). The relation between soil water retention and particle size distribution parameters for some predominantly sandy Western Australian soils. Australian Journal of Soil Research, 34(5), 695

Tyree MT, Yang S. (1990). Water-storage capacity of Thuja, Tsuga and Acer stems measured by dehydration isotherms. The contribution of capillary water and cavitation. Planta,182, 420-426.

\section{APPENDIX 1}

Table 1. Hydraulic parameters used in simulations.

\begin{tabular}{|c|c|c|c|}
\hline Parameter & Value & Units & Description \\
\hline $\mathrm{k}_{\mathrm{u}}$ & 56 & $\mathrm{~L} \mathrm{~h}^{-1} \mathrm{MPa}^{-1}$ & Conductivity through root cortex \\
\hline $\mathrm{k}_{\mathrm{r}}$ & 380 & $\mathrm{~L} \mathrm{~h}^{-1} \mathrm{MPa}^{-1}$ & Conductivity along xylem conducting elements of a root \\
\hline $\mathrm{k}_{\mathrm{t}}$ & 1125 & $\mathrm{~L} \mathrm{~h}^{-1} \mathrm{MPa}^{-1}$ & Conductivity along xylem conducting elements of the trunk \\
\hline $\mathrm{k}_{\mathrm{p}}$ & 112 & $\mathrm{~L} \mathrm{~h}^{-1} \mathrm{MPa}^{-1}$ & Conductivity along xylem conducting elements of petiole and leaf veins \\
\hline $\mathrm{c}_{\mathrm{r}}$ & 0.01 & $\mathrm{~m}^{3} \mathrm{~m}^{-3} \mathrm{MPa}^{-1}$ & Specific capacitivity of main roots \\
\hline $\mathrm{c}_{\mathrm{t}}$ & 0.01 & $\mathrm{~m}^{3} \mathrm{~m}^{-3} \mathrm{MPa}^{-1}$ & Specific capacitivity of the trunk \\
\hline$\tau_{\mathrm{l}}$ & 0.02 & $\mathrm{~m}^{3} \mathrm{~m}^{-3} \mathrm{MPa}^{-1}$ & Specific capacitivity of leaves \\
\hline$\tau_{C}$ & 2000 & $\mathrm{~s}$ & Characteristic time for stomatal opening \\
\hline & 800 & $\mathrm{~s}$ & Characteristic time for stomatal closing \\
\hline
\end{tabular}

